\title{
Long-term outcomes of different bortezomib- based regimens in Chinese myeloma patients
}

This article was published in the following Dove Press journal:

OncoTargets and Therapy

28 January 2016

Number of times this article has been viewed

\section{Hua Wang ${ }^{1-3, *}$ \\ Liang Wang ${ }^{1-3, *}$ \\ Yue $\mathrm{Lu}^{\mathrm{I}-3}$ \\ Xiaoqin Chen ${ }^{1-3}$ \\ Qirong Gengl-3 \\ Weida Wang ${ }^{1-3}$ \\ Zhongjun $\mathrm{Xia}^{1-3}$}

'Department of Hematological Oncology, ${ }^{2}$ State Key Laboratory of Oncology in South China, Sun Yat-Sen University Cancer Center, Guangzhou, ${ }^{3}$ Collaborative Innovation Center for Cancer Medicine, Guangzhou, Guangdong, People's Republic of China

*These authors contributed equally to this work
Correspondence: Zhongjun Xia Department of Hematological Oncology, Sun Yat-Sen University Cancer Center, 65I Dong Feng Road East, Guangzhou, Guangdong 510060, People's Republic of China

Tel +86 2087343797

Fax +86 2087363019

Email zhongjunxia_15@sina.com

\begin{abstract}
Bortezomib has significantly increased the response rates in multiple myeloma (MM), but optimal bortezomib-based regimens for initial MM therapy have not yet been defined. We retrospectively compared the outcomes of 128 patients newly diagnosed with symptomatic MM who received either bortezomib combined with dexamethasone (PD) or three-drug combinations of PD with liposomal doxorubicin (PAD) or thalidomide (PTD). The overall response rate (ORR), very good partial response (VGPR) rate, and complete remission $\mathrm{CR} /$ near-complete remission (nCR) results were better for the $\mathrm{PAD}$ and $\mathrm{PTD}$ regimens than for the PD group. Three-year overall survival (OS) was $80.1 \%, 72.5 \%$, and $61.8 \%$ with PAD, PTD, and $\mathrm{PD}$ regimens, respectively. The 3-year OS rate of PAD and PTD was significantly higher than that of $\mathrm{PD}(80.1 \%$ vs $61.8 \%, P=0.024 ; 72.5 \%$ vs $61.8 \%, P=0.035)$, but the difference was not statistically significant between PAD and PTD $(80.1 \%$ vs $72.5 \%, P=0.843)$. Similarly, the $\mathrm{PAD}$ and PTD regimens resulted in significantly superior 3-year progression-free survival (PFS) rates. The patients in the PTD arm were more frequently observed with grade $1-3$ peripheral neuropathy (PN), compared to those in the PAD and PD groups, especially grade 2-3 PN. PN developed less frequently without sacrificing the efficacy when bortezomib was administered subcutaneously rather than intravenously. Our experience suggests that the three-drug combinations PAD and PTD produce a better outcome than PD, especially with respect to PAD, with fewer adverse events.
\end{abstract}

Keywords: multiple myeloma, bortezomib, prognostic factors, peripheral neuropathy, subcutaneous

\section{Introduction}

Multiple myeloma (MM) is a fatal plasma cell malignancy that mainly affects older individuals. ${ }^{1}$ Before the advent of novel therapies, conventional chemotherapy resulted in a complete remission (CR) rate of 5\%-8\% and median survival of about 3-5 years in MM patients. ${ }^{2}$ The outcome of MM has significantly improved due to the advances in treatment options. Bortezomib, which is a reversible proteasome inhibitor, has been shown to have a significant antitumor effect through various kinds of mechanisms. Multiple randomized controlled clinical trials confirm that the introduction of bortezomib in frontline regimens has increased the response rates and depth of responses, with even better survival time than using conventional chemotherapy regimens, such as melphalan and prednisone (MP) or vincristine, doxorubicin, and dexamethasone (VAD). ${ }^{3-5}$ Currently, combination chemotherapy based on bortezomib contains two or three drugs, but the differences between various kinds of regimens in terms of the effective rates and long-term outcomes have not been fully defined yet because there are few studies referring to these. Especially, the data on this topic from the People's Republic of China are not sufficient. Since 2006, a fraction of patients newly diagnosed with MM were treated with different chemotherapy regimens based on bortezomib 
at Sun Yat-Sen University Cancer Center. In this study, we report the results of our retrospective analysis, the aim of which was to compare the effective rates and therapeutic outcomes of different combination therapies based on bortezomib for MM.

\section{Materials and methods \\ Patients}

From June 2006 to January 2014, a total of 128 consecutive patients newly diagnosed with MM received combination therapies based on bortezomib as the firstline chemotherapy for at least two cycles at the SunYat-Sen University Cancer Center. These patients were identified through the hospital discharge registry system and electronic medical records. The inclusion criteria of this retrospective clinical study were as follows: 1) newly diagnosed with symptomatic MM based on the diagnostic criteria of the World Health Organization; 2) had measurable monoclonal protein (M protein) in blood or urine; 3) previously untreated patients; 4) no previous or concomitant tumor; and 5) complete clinical information and long-term follow-up data available. All patients were staged according to the International Staging System (ISS) and Durie-Salmon (DS) staging. The Institutional Review Board of Sun Yat-Sen University Cancer Center approved this study, and all patients provided written informed consent forms in compliance with institutional guidelines. The study was performed in accordance with the guidelines of the Declaration of Helsinki.

\section{Treatment}

Bortezomib-based combination chemotherapy was given to patients, repeated every 3 weeks or 4 weeks as follows: 1) $\mathrm{PD}$ (bortezomib and dexamethasone); 2) PAD (bortezomib, dexamethasone, and liposomal doxorubicin); and 3) PTD (bortezomib, dexamethasone, and thalidomide). Among the treatment protocols, bortezomib $\left(1.3 \mathrm{mg} / \mathrm{m}^{2}\right)$ and dexamethasone $(40 \mathrm{mg} / \mathrm{d})$ were administered intravenously on Days 1, 4, 8, and 11, while liposomal doxorubicin $\left(40 \mathrm{mg} / \mathrm{m}^{2}\right)$ was administered intravenously on Day 1 , and thalidomide (100 mg) was given orally each day, repeated every 3 weeks. After 2012, the protocol was amended to reduce the incidence of peripheral neuropathy. The administration methods for bortezomib were modified to subcutaneous (SC) injection $\left(1.3 \mathrm{mg} / \mathrm{m}^{2}\right)$ on Days $1,8,15$, and 22 , repeated every 4 weeks. Similarly, dexamethasone $(40 \mathrm{mg} / \mathrm{d})$ was given intravenously on the same day of the week.

Sixty-four patients underwent autologous hematopoietic stem cell transplantation (AHSCT) after induction therapy, including 22 cases who received PAD induction therapy, 27 cases who received PTD, and 15 cases who received PD. Patients with age $\geq 65$ years or organ dysfunction were excluded from receiving AHSCT. After induction therapy or AHSCT, thalidomide (100 mg/d) was given orally to patients as maintenance treatment until disease progression or until they could not endure adverse drug reactions. Aspirin was routinely used to prevent thrombosis in patients who received PTD induction therapy or thalidomide for maintenance treatment. Granulocyte colony-stimulating factor (G-CSF) ( $5 \mu \mathrm{g} / \mathrm{kg} / \mathrm{d}, \mathrm{SC}$ injection) was administered to patients whose peripheral white blood cell counts were $<2.0 \times 10^{9} / \mathrm{L}$; G-CSF was administered until neutrophil counts recovered. Chemotherapy was administered only if the absolute neutrophil count was $>1.5 \times 10^{9} / \mathrm{L}$ and the platelet count was $>75 \times 10^{9} / \mathrm{L}$ before each cycle.

\section{Response and toxicity criteria}

The treatment response was assessed after every cycle during induction therapy and at 3 months and 6 months after AHSCT, in accordance with the International Melanoma Working Group response criteria. CR was defined as negative serum/urine immunofixation and normal bone marrow morphology. Very good partial response (VGPR) required $>90 \%$ decrease of serum monoclonal proteins (M protein) and light chain in urine $<100 \mathrm{mg} / 24$ hours. During the maintenance treatment period, patients were followed up and assessed by their oncologist in the outpatient department every 2 months. All adverse effects were evaluated at each visit and graded based on the National Cancer Institute Common Terminology Criteria of Adverse Events v3.0.

\section{Statistical analysis}

Overall survival (OS) was calculated from the time of diagnosis until death from any cause or until the time of the last follow-up visit for the surviving patients. Progression-free survival (PFS) was defined as the interval from the initiation of chemotherapy to the time of the first documented disease progression or relapse or the interval from chemotherapy initiation to the last follow-up visit. The chi-square test was used to calculate statistical group comparisons of categorical variables. Survival analysis was performed using the KaplanMeier method, and comparisons were calculated using the log-rank test. Multivariate analysis was used to estimate the prognostic impact of different variables in terms of OS and PFS using the Cox regression model. The variables that were included in a multivariate analysis were statistically significant by univariate analysis. $P<0.05$ was considered 
statistically significant, and all $P$-values correspond to two-sided significance tests. Statistical analyses were performed using SPSS 17.0 software.

\section{Results}

\section{Characteristics of patients}

The comparative patient baseline characteristics for the PAD, PTD, and PD groups are summarized in Table 1. The median age at diagnosis was 60 years (range: $31-83$ years) for the PAD group, 63 years (range: 29-79 years) for the PTD group, and 61 years (range: $38-81$ years) for the PD group. According to DS staging, 100 (78.1\%) patients were in stage III, while 96 (75\%) patients were in stages II and III according to ISS staging. Among the total of 128 patients, 70 received bortezomib by SC administration and 58 by intravenous (IV) administration. As shown in Table 1, there were no significant differences in the rates of these characteristics between the three treatment groups.

\section{Response}

Totally, 164 cycles (median of four cycles) for the PAD group, 248 cycles (median of four cycles) for PTD group, and 156 cycles (median of four cycles) for PD group were performed. Table 2 summarizes the response rates for every treatment group. Postinduction overall response rate (ORR; $\geq$ partial response [PR]) in all the 128 eligible patients was $81.3 \%$, including $28.1 \%$ VGPR and $32.1 \% \mathrm{CR} /$ near-complete remission (nCR) rates. The ORR rates for the PAD or PTD group were significantly higher than those in the PD group $(87.5 \%$ and $86.5 \%$, vs $66.7 \%$, respectively; $P=0.030$ and 0.026 ). The response rate in terms of VGPR plus $\mathrm{CR} / \mathrm{nCR}$ rates in all the patients was $60.2 \%$. VGPR rates in PAD and PTD groups were significantly higher than that in the PD group (35.0\% and $32.7 \%$, vs $13.9 \%$, respectively; $P=0.034$ and 0.045 ). Similarly, $\mathrm{CR} / \mathrm{nCR}$ rates in $\mathrm{PAD}$ and PTD arms were superior to that in PD $(40.0 \%$ and $36.5 \%$, vs $16.7 \%$, respectively; $P=0.025$ and 0.042 ). Of the whole

Table I Baseline characteristics of patients

\begin{tabular}{|c|c|c|c|c|}
\hline \multirow[t]{2}{*}{ Characteristic } & \multirow{2}{*}{$\begin{array}{l}\text { Total }(n=128) \\
N(\%)\end{array}$} & \multirow{2}{*}{$\frac{\text { PAD }(n=40)}{N(\%)}$} & \multirow{2}{*}{$\frac{\text { PTD }(n=52)}{N(\%)}$} & \multirow{2}{*}{$\frac{P D(n=36)}{N(\%)}$} \\
\hline & & & & \\
\hline \multicolumn{5}{|l|}{ Age, years } \\
\hline$<65$ & $82(64.1)$ & $25(62.5)$ & $34(65.4)$ & $23(63.9)$ \\
\hline$\geq 65$ & $46(35.9)$ & $15(37.5)$ & $18(34.6)$ & $13(36.1)$ \\
\hline Sex, male & $84(65.6)$ & $28(70.0)$ & $33(63.5)$ & $23(63.9)$ \\
\hline \multicolumn{5}{|l|}{ ECOG PS } \\
\hline $0-1$ & $110(85.9)$ & $33(82.5)$ & $47(90.4)$ & $30(83.3)$ \\
\hline$\geq 2$ & $18(14.1)$ & $7(17.5)$ & $5(9.6)$ & $6(16.7)$ \\
\hline \multicolumn{5}{|c|}{ Durie-Salmon stage } \\
\hline $\mathrm{IA}$ & $7(5.5)$ & $2(5.0)$ & $3(5.8)$ & $2(5.6)$ \\
\hline $2 \mathrm{~A}$ & $21(16.4)$ & $5(12.5)$ & $9(17.3)$ & $7(19.4)$ \\
\hline $3 \mathrm{~A}$ & $67(52.3)$ & $21(52.5)$ & $28(53.8)$ & $18(50.0)$ \\
\hline $3 B$ & $33(25.8)$ & $12(30.0)$ & $12(23.1)$ & $9(25.0)$ \\
\hline \multicolumn{5}{|l|}{ ISS stage } \\
\hline 1 & $32(25.0)$ & $14(35.0)$ & II (2।.2) & $7(19.4)$ \\
\hline II & $50(39.1)$ & $9(22.5)$ & $23(44.2)$ & $18(50.0)$ \\
\hline III & $46(35.9)$ & $17(42.5)$ & $18(34.6)$ & II (30.6) \\
\hline \multicolumn{5}{|l|}{ Type of myeloma } \\
\hline $\lg A$ & $30(23.4)$ & $8(20.0)$ & 14 (26.9) & $8(22.2)$ \\
\hline $\lg G$ & $65(50.8)$ & $26(65.0)$ & $23(44.3)$ & $16(44.4)$ \\
\hline $\lg D$ & $4(3.1)$ & $\mathrm{I}(2.5)$ & I (1.9) & $2(5.6)$ \\
\hline Light chain & $29(22.7)$ & $5(12.5)$ & $14(26.9)$ & $10(27.8)$ \\
\hline \multicolumn{5}{|l|}{ Serum LDH } \\
\hline$\leq \mathrm{ULN}$ & $107(83.6)$ & $32(80.0)$ & $43(82.7)$ & $32(88.9)$ \\
\hline$>$ ULN & $21(16.4)$ & $8(20.0)$ & $9(I 7.3)$ & $4(11.1)$ \\
\hline \multicolumn{5}{|c|}{ Genetic abnormalities } \\
\hline Yes $^{\mathrm{a}}$ & 71 (55.5) & $24(60.0)$ & $29(55.8)$ & $18(50.0)$ \\
\hline No & $57(44.5)$ & $16(40.0)$ & $23(44.2)$ & $18(50.0)$ \\
\hline \multicolumn{5}{|c|}{ Administration methods } \\
\hline Subcutaneous & $70(54.7)$ & $23(57.5)$ & $34(65.4)$ & $13(36.1)$ \\
\hline Intravenous & $58(45.3)$ & $17(42.5)$ & $18(34.6)$ & $23(63.9)$ \\
\hline
\end{tabular}

Note: aPatients with abnormalities of $|3 q| 4$, Iq2I, $14 q 32$, and $17 p \mid 3$

Abbreviations: ECOG PS, Eastern Cooperative Oncology Group performance status; Ig, immunoglobulin; ISS, International Staging System; LDH, lactate dehydrogenase; PAD, PD with liposomal doxorubicin; PD, bortezomib combined with dexamethasone; PTD, PD with thalidomide; ULN, upper limit of normal. 
Table 2 Postinduction overall response rates of regimens

\begin{tabular}{|c|c|c|c|c|c|c|c|c|}
\hline & \multicolumn{2}{|l|}{ ORR } & \multicolumn{2}{|l|}{ PR } & \multicolumn{2}{|l|}{ VGPR } & \multicolumn{2}{|l|}{ CR/nCR } \\
\hline & $\mathbf{N}(\%)$ & $P$-value ${ }^{a}$ & $\mathbf{N}(\%)$ & $P$-value & $\mathbf{N}(\%)$ & $P$-value ${ }^{b}$ & $\mathbf{N}(\%)$ & $P$-value ${ }^{c}$ \\
\hline PAD & $35(87.5)$ & 0.030 & $5(12.5)$ & 0.016 & $14(35.0)$ & 0.034 & $16(40.0)$ & 0.025 \\
\hline PTD & $45(86.5)$ & 0.026 & $9(17.3)$ & 0.045 & 17 (32.7) & 0.045 & $19(36.5)$ & 0.042 \\
\hline PD & $24(66.7)$ & & $13(36.1)$ & & $5(13.9)$ & & $6(16.7)$ & \\
\hline
\end{tabular}

Notes: aPatients who received PAD or PTD demonstrated significantly higher ORR compared to those on PD $(87.5 \%$ and $86.5 \%$ vs $66.7 \%$, respectively; $P=0.030$ and 0.026 respectively). ${ }^{\text {} R}$ Response rates defined as VGPR were significantly higher for PAD and PTD than for PD ( $35.0 \%$ and $32.7 \%$, vs $13.9 \%$, respectively; $P=0.034$ and 0.045 respectively). 'Rates of patients showing CR/nCR were higher in PAD and PTD than in PD ( $40.0 \%$ and $36.5 \%$, vs $16.7 \%$, respectively; $P=0.025$ and 0.042 respectively).

Abbreviations: $\mathrm{CR} / \mathrm{nCR}$, complete remission/near-complete remission; ORR, overall response rate; PAD, PD with liposomal doxorubicin; PD, bortezomib combined with dexamethasone; PR, partial response; PTD, PD with thalidomide; VGPR, very good partial response.

group of 128 patients, the overall $\mathrm{CR} / \mathrm{nCR}$ rate and VGPR rates were $43.0 \%$ and $71.1 \%$ after AHSCT. Posttransplantation, $\mathrm{CR} / \mathrm{nCR}$ and at least VGPR rates remained significantly higher in both PAD and PTD groups than in the PD group in transplant-eligible patients.

\section{Treatment outcomes and prognosis}

The median follow-up time for the 128 patients from the time of diagnosis was 24 months (4.0-66.0 months). The median PFS and OS for all the patients were 38 months $(95 \%$ confidence interval: 31.9-44.1 months) (Figure 1A) and not reached at 66 months (Figure 1B), respectively. The rates of 3-year PFS were 55.6\%, 64.6\%, and 32.9\%, respectively, for the PAD, PTD, and PD regimens. The 3-year PFS rate for PAD and PTD was significantly higher than that for PD $(55.6 \%$ and $64.6 \%$ vs $32.9 \%$, respectively; $P=0.020$ and 0.024 , respectively; Figure 2A). But, no significant difference was found between PAD and PTD groups. The rates of 3-year OS were $80.1 \%, 72.5 \%$, and $61.8 \%$, respectively, with PAD, PTD, and PD regimens. The 3-year OS rates of $\mathrm{PAD}$ and $\mathrm{PTD}$ were significantly higher than that of $\operatorname{PD}(P=0.024$ and 0.035 , respectively), but the difference was not statistically significant between PAD and PTD $(P=0.843)$ (Figure 2B). No significant survival differences were found between the patients who received bortezomib by SC and those receiving it by IV (Figure 3). Upon further analysis, there were no significant survival differences observed according to the administration methods for bortezomib in every subgroup.

The results of the multivariate analyses of clinical variables considered to be predictors of PFS and OS are shown in Table 3. Multivariate analysis revealed that a higher DS stage, elevated lactate dehydrogenase (LDH) level, induction therapy regimens, and certain cytogenetic
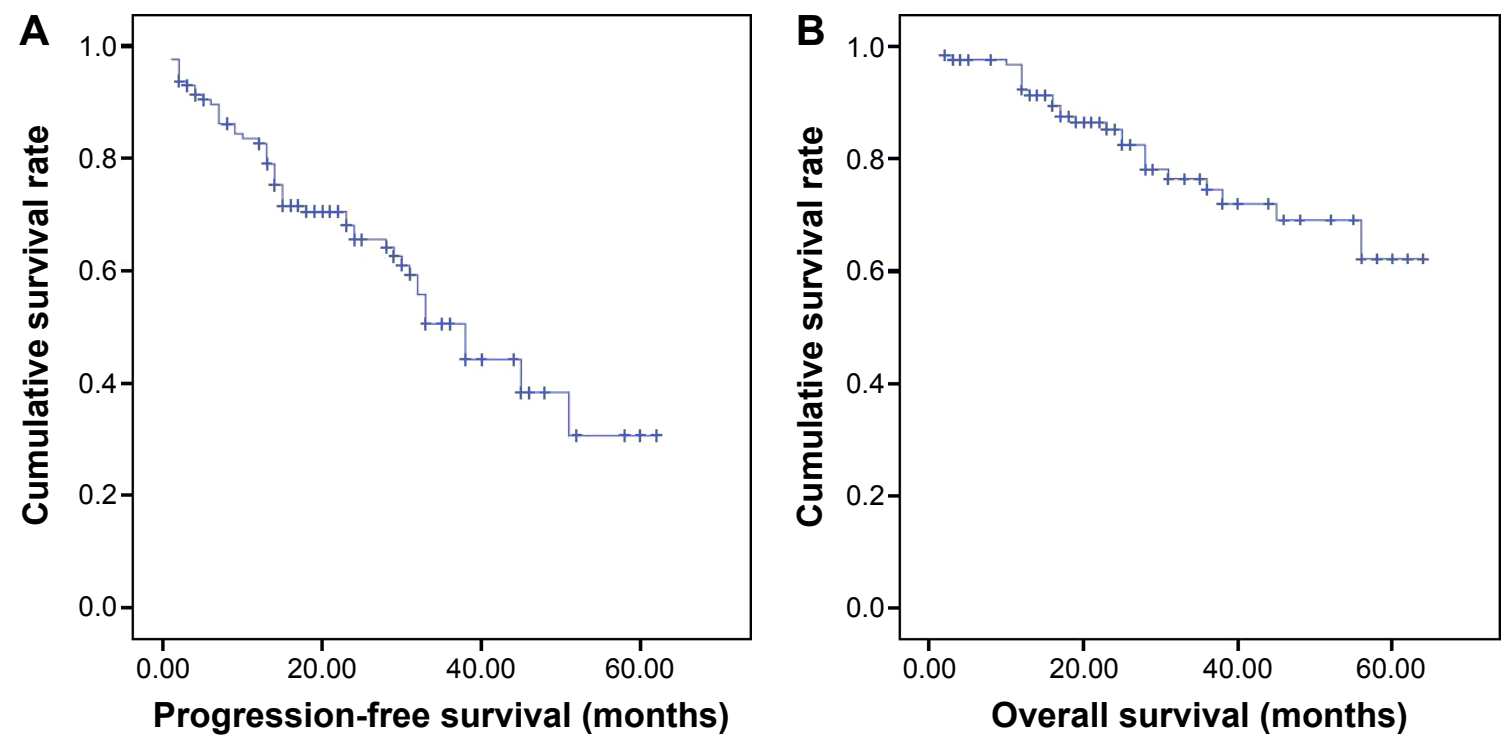

$\curvearrowleft$ Survival function + Censored

Figure I The survival data for all patients.

Notes: (A) Progression-free survival. (B) Overall survival. 

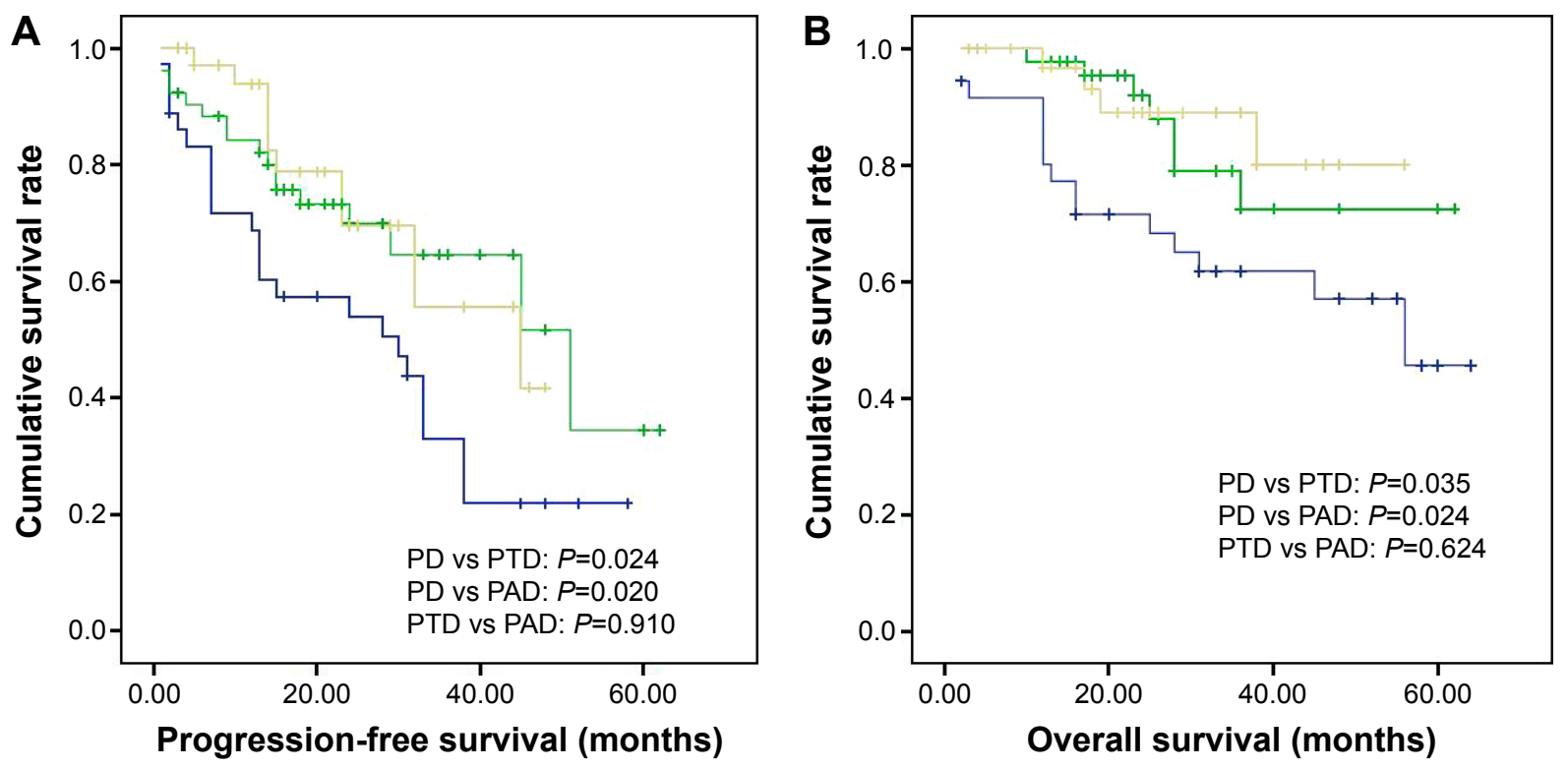

\section{Chemotherapy regimens

$\neg$ PD $\neg$ PTD $\neg$ PAD + PD-censored +PTD-censored + PAD-censored

Figure 2 Survival curves for patients with multiple myeloma.

Notes: (A) The 3-year PFS rates for PAD, PTD, and PD groups were 55.6\%, 64.6\%, and 32.9\%, respectively; (B) 3-year OS rates for PAD, PTD, and PD groups were $89 \%$, $72.5 \%$, and $61.8 \%$, respectively.

Abbreviations: OS, overall survival; PAD, PD with liposomal doxorubicin; PD, bortezomib combined with dexamethasone; PTD, PD with thalidomide; PFS, progressionfree survival.
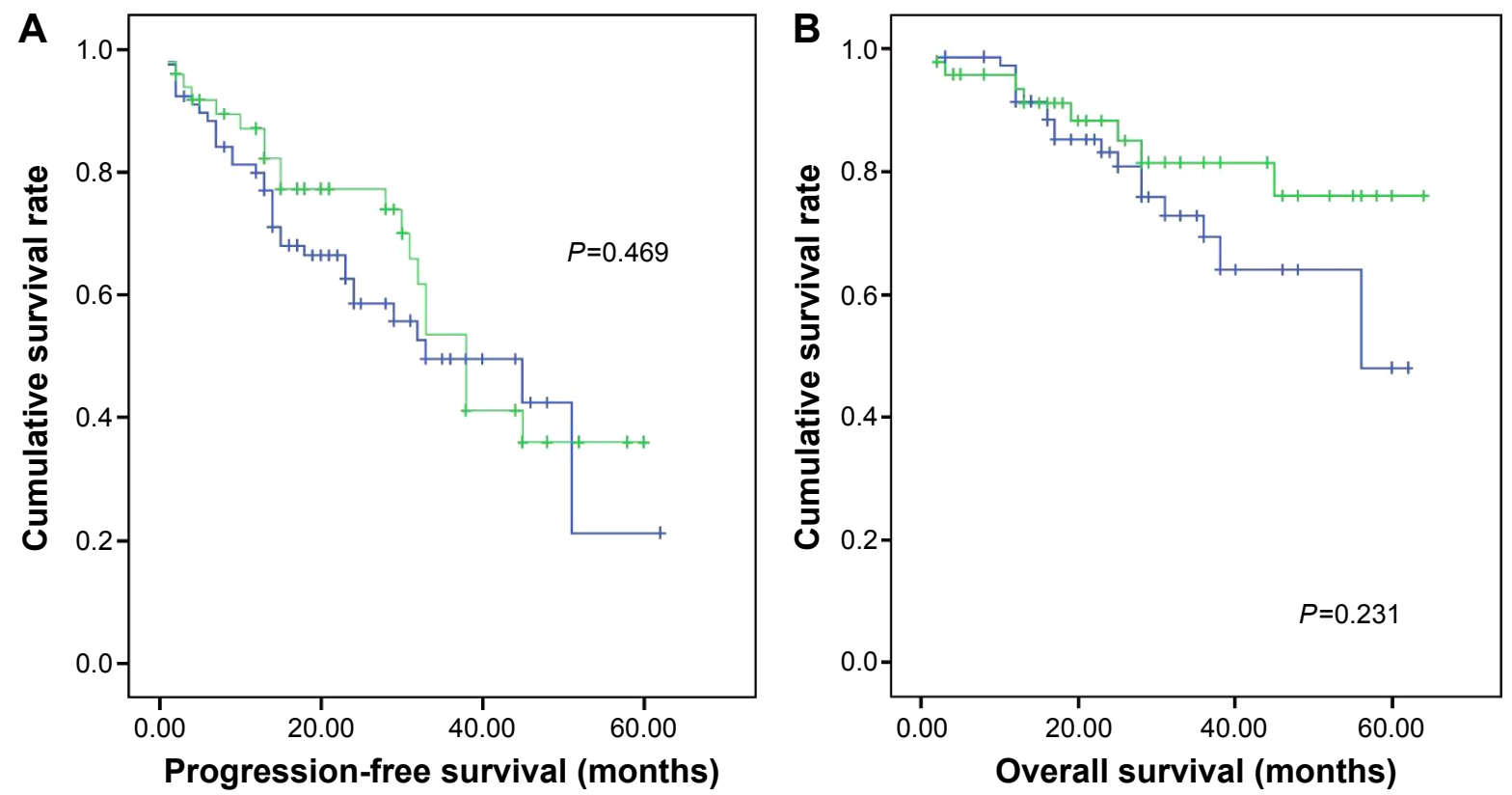

\section{Administration methods \\ $\neg \mathrm{SC}-$ IV + SC-censored + IV-censored}

Figure 3 Survival outcomes of patients based on the methods of administration of bortezomib.

Notes: (A) PFS of patients according to the administration methods of bortezomib (SC vs IV). (B) OS of patients according to the administration methods for bortezomib (SC vs IV).

Abbreviations: IV, intravenous; OS, overall survival; PFS, progression-free survival; SC, subcutaneous. 
Table 3 Multivariate analysis of risk factors for PFS and OS

\begin{tabular}{|c|c|c|c|c|c|c|}
\hline \multirow[t]{2}{*}{ Risk factor } & \multicolumn{2}{|l|}{ PFS } & \multirow[t]{2}{*}{$P$-value } & \multicolumn{2}{|l|}{ OS } & \multirow[t]{2}{*}{ P-value } \\
\hline & HR & $95 \% \mathrm{Cl}$ & & HR & $95 \% \mathrm{Cl}$ & \\
\hline Age $\geq 65$ years & 1.287 & $0.508-3.258$ & 0.594 & 2.531 & $0.483-13.254$ & 0.272 \\
\hline ECOG PS $\geq 2$ & 0.878 & $0.422-1.827$ & 0.727 & I.73। & $0.632-4.743$ & 0.286 \\
\hline Durie-Salmon stage & 2.026 & $1.366-3.210$ & 0.001 & 3.528 & $1.47 \mid-8.458$ & 0.005 \\
\hline ISS stage & 1.104 & $0.625-1.949$ & 0.733 & 1.522 & $0.576-4.023$ & 0.397 \\
\hline LDH $>$ ULN & 2.830 & I.279-5.322 & 0.008 & 6.238 & $2.193-17.750$ & 0.001 \\
\hline Regimens $^{\mathrm{a}}$ & 0.580 & $0.426-0.874$ & 0.007 & 0.422 & $0.236-0.754$ & 0.004 \\
\hline $\mathrm{FISH}^{\mathrm{b}}$ & $\mathrm{I} .4 \mathrm{I} \mathrm{I}$ & $0.736-2.705$ & 0.299 & 3.778 & I.327-10.756 & 0.013 \\
\hline
\end{tabular}

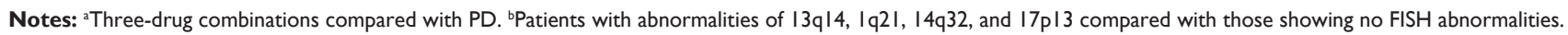
Abbreviations: $\mathrm{Cl}$, confidence interval; ECOG PS, Eastern Cooperative Oncology Group performance status; FISH, interphase fluorescence in situ hybridization; HR, hazard ratio; ISS, International Staging System; LDH, lactate dehydrogenase; OS, overall survival; PFS, progression-free survival; ULN, upper limit of normal; PD, bortezomib combined with dexamethasone.

abnormalities significantly affected patients' PFS and OS, whereas age, Eastern Cooperative Oncology Group performance status (ECOG PS), and ISS stage had no effect on PFS and OS.

\section{Toxicity}

In every treatment group, no patient had to discontinue treatment for severe adverse events and no treatmentrelated deaths occurred. The main hematological toxicities (Grade 3/4) were thrombocytopenia (16.4\%), neutropenia (14.8\%), and anemia (7.9\%). Among the patients in each subgroup, no significant difference was found in terms of hematological toxicities (Grade 3/4). The most frequently observed nonhematological toxicities (all grades) included peripheral neuropathy $(\mathrm{PN})(48.4 \%)$, infection (25.8\%), fatigue $(25.0 \%)$, etc (Table 4$)$. The occurrence rates of constipation and herpes zoster in the PTD arm were significantly higher than in the PAD and PD groups. In all the patients enrolled, no one was observed with grade $4 \mathrm{PN}$. The patients in the PTD arm were more frequently observed with grade 1-3 PN compared to those in the PAD and PD groups. The incidence of PN (grades 2-3) in the PTD group was significantly higher compared to that in the PAD and PD arms. For all patients, the incidence of grades 2-3 PN was significantly higher in the IV administration group than in the SC administration group (Table 5). Similarly, the incidence of grades 2-3 PN was significantly different according to the administration methods for bortezomib in the PAD and PTD groups (Table 5). In the PD group, the incidence of grades 2-3 PN after IV administration was higher, although the difference was not statistically significant relative to the $\mathrm{SC}$ administration group.

Table 4 Treatment-related adverse events

\begin{tabular}{|c|c|c|c|c|}
\hline \multirow[t]{2}{*}{ Adverse events } & Total, n (\%) & PAD, n (\%) & PTD, n (\%) & PD, n (\%) \\
\hline & $(n=128)$ & $(n=40)$ & $(n=52)$ & $(n=36)$ \\
\hline \multicolumn{5}{|l|}{ Hematologic events (Grade 3/4) } \\
\hline Neutropenia & $19(14.8)$ & $7(17.5)$ & $8(15.4)$ & $4(I I . I)$ \\
\hline Thrombocytopenia & $21(16.4)$ & $8(20.0)$ & $8(15.4)$ & $5(13.9)$ \\
\hline Anemia & $13(7.9)$ & $5(12.5)$ & $6(11.5)$ & $2(5.6)$ \\
\hline \multicolumn{5}{|c|}{ Nonhematologic events (all grades) } \\
\hline Fatigue & $32(25.0)$ & $12(30.0)$ & $13(25.0)$ & $7(19.4)$ \\
\hline Infection & $33(25.8)$ & $13(32.5)$ & $13(25.0)$ & $7(19.4)$ \\
\hline Constipation ${ }^{\mathrm{a}}$ & $28(21.9)$ & $5(12.5)$ & $21(40.4)$ & $2(5.6)$ \\
\hline Diarrhea & $16(12.5)$ & $5(12.5)$ & $6(11.5)$ & $5(13.9)$ \\
\hline Pleural effusion and ascites & $7(5.5)$ & $2(5.0)$ & $3(5.8)$ & $2(5.6)$ \\
\hline Herpes zoster ${ }^{\mathrm{b}}$ & $20(15.6)$ & $3(7.5)$ & $15(28.8)$ & $2(5.6)$ \\
\hline Deep vein thrombosis & $\mathrm{I}(0.8)$ & $0(0.0)$ & $\mathrm{I}(\mathrm{l} .9)$ & $0(0.0)$ \\
\hline Peripheral neuropathy ${ }^{c}$ & $62(48.4)$ & $15(37.5)$ & $34(65.3)$ & $13(36.1)$ \\
\hline Grade I & $40(31.3)$ & II (27.5) & $19(36.5)$ & $10(27.8)$ \\
\hline Grade $2 / 3^{d}$ & $22(17.1)$ & $4(10.0)$ & $15(28.8)$ & $3(8.3)$ \\
\hline
\end{tabular}

Notes: alncidence of constipation for the PTD arm was significantly higher than for the PAD and PD groups $\left(P=0.003\right.$ and $<0.00$ I, respectively). ${ }^{b}$ Incidence of herpes zoster for the PTD arm was significantly higher than for the PAD and PD groups $(P=0.01 \mathrm{I}$ and 0.007 , respectively). 'Peripheral neuropathy of all grades was more frequently reported in patients in the PTD group compared to those in the other groups, which was obviously higher than that of the PAD and PD groups ( $P=0.008$ and 0.007 , respectively). Incidence of grades 2-3 peripheral neuropathy for the PTD arm was significantly higher than in the PAD and PD groups $(P=0.027$ and 0.019 , respectively). There was no significant difference in other treatment-related adverse events among groups.

Abbreviations: PAD, PD with liposomal doxorubicin; PD, bortezomib combined with dexamethasone; PTD, PD with thalidomide. 
Table 5 Peripheral neuropathy in patients according to the administration method of bortezomib

\begin{tabular}{|c|c|c|c|c|c|c|}
\hline & Subcutaneous & Intravenous & $P$-value & Subcutaneous & Intravenous & $P$-value \\
\hline & $\overline{\text { Grade I, n (\%) }}$ & $\overline{\text { Grade I, n (\%) }}$ & & $\overline{\text { Grade } 2 / 3, \text { n (\%) }}$ & $\overline{\text { Grade } 2 / 3, \mathrm{n}(\%)}$ & \\
\hline Total & $21(30.0)$ & $19(32.8)$ & 0.737 & $6(8.6)$ & $16(27.6)$ & 0.005 \\
\hline PAD & $6(26.1)$ & $5(29.4)$ & 0.816 & $0(0.0)$ & $4(23.5)$ & 0.014 \\
\hline PTD & $12(35.3)$ & $7(38.9)$ & 0.798 & $6(17.6)$ & $9(50.0)$ & 0.014 \\
\hline PD & $3(23.1)$ & $7(30.4)$ & 0.636 & $0(0.0)$ & $3(13.0)$ & 0.174 \\
\hline
\end{tabular}

Abbreviations: PAD, PD with liposomal doxorubicin; PD, bortezomib combined with dexamethasone; PTD, PD with thalidomide.

\section{Discussion}

MM still remains an incurable disease and nearly all patients eventually relapse and succumb to MM. To get a deeper response for longer duration is necessary for disease control, regardless of whether the patients are newly diagnosed ones or relapse/refractory patients. ${ }^{6-11}$ With the advent of novel proteasome inhibitors and immunomodulatory drugs, most patients can show higher response rate and improved PFS. Over the past few years, a number of prospective randomized clinical trials have demonstrated that the efficacies of bortezomib- and lenalidomide-based regimens are significantly superior to that of the traditional methods. ${ }^{3,12}$ However, few clinical trials have compared different regimens based on bortezomib to elucidate which is the most suitable one for the initial treatment of MM, with the aim of improving the long-term survival and quality of life of the patient. ${ }^{13,14}$ The objective of our study was to investigate the response rates, treatment outcomes, and toxicity profiles of different bortezomib-based regimens in Chinese myeloma patients. In this study, we found that the response rates for the PAD and PTD regimens were higher than those for PD, which could translate into improved survival.

In our study, the ORR for patients who were treated with the PD regimen was $66.7 \%$, including $16.7 \%$ with $\mathrm{CR} / \mathrm{nCR}$ and $13.9 \%$ with VGPR, which is consistent with the study conducted by He et al, ${ }^{15}$ also from the People's Republic of China. Actually, many studies showed that the ORRs were in the range of $60 \%-85 \%$ with the $\mathrm{PD}$ regimen, including at least $30 \%-40 \%$ VGPR and $\sim 20 \% \mathrm{CR} / \mathrm{nCR} .{ }^{12,13,16,17}$ The IFM 2005-01 study, ${ }^{12}$ a Phase III clinical trial showed that the $\mathrm{ORR}, \mathrm{CR} / \mathrm{nCR}$, and at least $\mathrm{VGPR}$ for the $\mathrm{PD}$ regimen were $78.5 \%, 14.8 \%$, and $37.7 \%$, respectively, in newly diagnosed patients regardless of adverse cytogenetic abnormalities or disease stage. $^{12}$

The PD regimen after combining with another drug, an immunomodulatory agent such as thalidomide or a conventional cytotoxic drug such as adriamycin, can yield ORR of $90 \%$, including at least $60 \%-70 \% \mathrm{VGPR}$ and $40 \%-50 \% \mathrm{CR} /$ nCR. ${ }^{18-20}$ At Sun Yat-Sen University Cancer Center, are used to replace adriamycin with liposomal doxorubicin because of the lower cardiotoxicity. In our analysis, patients were treated with a median of four cycles, and $\sim 34.4 \%$ cases only received two or three cycles; the PAD regimen had similar effect as the PTD regimen. In terms of ORR, VGPR, and CR/nCR rates, PAD and PTD had advantage over PD. We found that similar results were obtained by another team from the People's Republic of China. ${ }^{15}$ However, in a randomized trial, ${ }^{21}$ the Intergroupe Francophone du Mye'lome (IFM) research group reported that the regimen bortezomib and thalidomide plus dexamethasone (VTD), including reduced doses of bortezomib and thalidomide, yields higher VGPR rates after induction therapy, as well as CR plus VGPR rates after AHSCT, compared with VD (bortezomib-dexamethasone). In our study, the rates of 3-year OS in the PAD and PTD groups were significantly higher than those in the PD group ( $P=0.024$ and 0.035 , respectively). Interestingly, a recently published study ${ }^{22}$ showed that PTD did not appear to offer an advantage over VD in transplantation-ineligible patients with myeloma treated in US community practice. Nevertheless, half the number of patients in our study received AHSCT, which may be the main reason for the difference in the research results. Certainly, the results in our study should be interpreted within its limitations because it is a retrospective study. Further prospective research should be conducted on patients of larger sample sizes.

Multivariate analysis also indicated that a higher DS stage, elevated LDH level, induction therapy regimens, and certain cytogenetic abnormalities were independent prognostic factors for PFS and OS, which is in accordance with those seen in other studies. ${ }^{23}$ However, ISS stage seems to be less helpful for predicting survival in our study; the results indicate that ISS had limitations when applied on MM patients in the People's Republic of China. Our study and the study conducted by He et $\mathrm{al}^{15}$ from the People's Republic of China achieved consistent results in this respect. Previous studies confirmed that $\mathrm{LDH}$ is an adverse prognostic factor for myeloma. Chim et $\mathrm{al}^{24}$ reported that $\mathrm{LDH}$ is an adverse prognostic factor independent of ISS in transplant-eligible myeloma patients receiving bortezomib-based induction regimens. Moreover, multivariate analysis including LDH, ISS 
stage, etc showed that ISS stage was no longer an independent prognostic factor.

Many studies show that combined treatments based on bortezomib do not seem to induce serious adverse reactions, and our results indicated this as well. ${ }^{3,12,13,16-20} \mathrm{PN}$ is another common adverse effect of bortezomib; however, it has dose-limiting and reversible toxicity. ${ }^{25,26}$ In this study, the incidence of PN was higher in the PTD group compared to the PAD and PD groups, but whether bortezomib plus thalidomide increased the incidence of PN and its severity was not clear. Several clinical trials have evaluated the benefit of prolonging the administration interval of bortezomib as once-weekly IV injection and have shown that this strategy can significantly reduce the incidence rate of $\mathrm{PN}$, without appearing to influence the efficacy. ${ }^{27,28}$ Similarly, the SC administration of bortezomib as twice-weekly injection was found to be noninferior in efficacy to standard twiceweekly IV administration, with an improved safety profile. ${ }^{29}$ Therefore, we began to give bortezomib by once-weekly $\mathrm{SC}$ injection to further reduce the incidence rate of PN. Our results showed that this strategy may reduce the incidence rate of $\mathrm{PN}$ without affecting efficacy. It was reported that the incidence rate of herpes zoster was 5\%-13\% in Europe and the US, but Asian patients had significantly higher incidence after treatments based on bortezomib..$^{30,31}$ In our study, herpes zoster was observed in $28.8 \%$ of cases in the PTD group without preventive antiviral use, which was much higher than in other groups.

\section{Conclusion}

In conclusion, the combined treatment with the three drugs is proven to be superior to bortezomib plus dexamethasone, and the PAD regimen is noninferior in efficacy, with fewer adverse events. According to the occurrence rate and degree of PN, PAD is superior to PTD. SC injection of bortezomib decreases the incidence of $\mathrm{PN}$ requiring medical treatment in Chinese patients without sacrificing the efficacy of bortezomib. Therefore, SC injection of bortezomib should be considered in Chinese MM patients.

\section{Acknowledgment}

The authors would like to thank the patients and their families, and all the investigators, including the physicians, nurses, and laboratory technicians who participated in this study.

\section{Disclosure}

The authors report no conflicts of interest in this work.

\section{References}

1. Smith D, Yong K. Multiple myeloma. BMJ. 2013;346:f3863.

2. Cavo M, Zamagni E, Tosi P, et al; Bologna 2002 study. Superiority of thalidomide and dexamethasone over vincristine-doxorubicindexamethasone (VAD) as primary therapy in preparation for autologous transplantation for multiple myeloma. Blood. 2005;106(1):35-39.

3. Sonneveld P, Schmidt-Wolf IG, van der Holt B, et al. Bortezomib induction and maintenance treatment in patients with newly diagnosed multiple myeloma: results of the randomized phase III HOVON-65/ GMMG-HD4 trial. J Clin Oncol. 2012;30(24):2946-2955.

4. Huang H, Zhou L, Peng L, Fu W, Zhang C, Hou J. Bortezomib-thalidomidebased regimens improved clinical outcomes without increasing toxicity as induction treatment for untreated multiple myeloma: a meta-analysis of phase III randomized controlled trials. Leuk Res. 2014;38(9): 1048-1054.

5. KouroukisTC,BaldassarreFG, HaynesAE,ImrieK, ReeceDE, Cheung MC. Bortezomib in multiple myeloma: systematic review and clinical considerations. Curr Oncol. 2014;21(4):e573-e603.

6. van de Velde HJ, Liu X, Chen G, Cakana A, Deraedt W, Bayssas M. Complete response correlates with long-term survival and progressionfree survival in high-dose therapy in multiple myeloma. Haematologica. 2007;92(10):1399-1406.

7. Niesvizky R, Richardson PG, Rajkumar SV, et al. The relationship between quality of response and clinical benefit for patients treated on the bortezomib arm of the international, randomized, phase 3 APEX trial in relapsed multiple myeloma. Br J Haematol. 2008;143(1):46-53.

8. Harousseau JL, Attal M, Avet-Loiseau H. The role of complete response in multiple myeloma. Blood. 2009;114(15):3139-3146.

9. Barlogie B, Anaissie E, Haessler J, et al. Complete remission sustained 3 years from treatment initiation is a powerful surrogate for extended survival in multiple myeloma. Cancer. 2008;113(2):355-359.

10. Harousseau JL, Avet-Loiseau H, Attal M, et al. Achievement of at least very good partial response is a simple and robust prognostic factor in patients with multiple myeloma treated with high-dose therapy: longterm analysis of the IFM 99-02 and 99-04 Trials. J Clin Oncol. 2009; 27(34):5720-5726.

11. Kim JS, Kim K, Cheong JW, et al; Korean Multiple Myeloma Working Party. Complete remission status before autologous stem cell transplantation is an important prognostic factor in patients with multiple myeloma undergoing upfront single autologous transplantation. Biol Blood Marrow Transplant. 2009;15(4):463-470.

12. Harousseau JL, Attal M, Avet-Loiseau H, et al. Bortezomib plus dexamethasone is superior to vincristine plus doxorubicin plus dexamethasone as induction treatment prior to autologous stem-cell transplantation in newly diagnosed multiple myeloma: results of the IFM 2005-01 phase III trial. J Clin Oncol. 2010;28(30):4621-4629.

13. Harousseau JL, Attal M, Leleu X, et al. Bortezomib plus dexamethasone as induction treatment prior to autologous stem cell transplantation in patients with newly diagnosed multiple myeloma: results of an IFM phase II study. Haematologica. 2006;91(11):1498-1505.

14. Rajkumar SV. Doublets, triplets, or quadruplets of novel agents in newly diagnosed myeloma? Hematology Am Soc Hematol Educ Program. 2012;2012:354-361.

15. He J, Yang L, Han X, et al. The choice of regimens based on bortezomib for patients with newly diagnosed multiple myeloma. PLoS One. 2014;9(6):e99174.

16. Rosinol L, Oriol A, Mateos MV, et al. Phase II PETHEMA trial of alternating bortezomib and dexamethasone as induction regimen before autologous stem-cell transplantation in younger patients with multiple myeloma: efficacy and clinical implications of tumor response kinetics. J Clin Oncol. 2007;25(28):4452-4458.

17. Jagannath S, Durie BG, Wolf JL, et al. Extended follow-up of a phase 2 trial of bortezomib alone and in combination with dexamethasone for the frontline treatment of multiple myeloma. Br J Haematol. 2009; 146(6):619-626. 
18. Palumbo A, Gay F, Falco P, et al. Bortezomib as induction before autologous transplantation, followed by lenalidomide as consolidationmaintenance in untreated multiple myeloma patients. $J$ Clin Oncol. 2010;28(5):800-807.

19. Cavo M, Tacchetti P, Patriarca F, et al; GIMEMA Italian Myeloma Network. Bortezomib with thalidomide plus dexamethasone compared with thalidomide plus dexamethasone as induction therapy before, and consolidation therapy after, double autologous stem-cell transplantation in newly diagnosed multiple myeloma: a randomised phase 3 study. Lancet. 2010;376(9758):2075-2085.

20. Mateos MV, Oriol A, Martinez-Lopez J, et al. Bortezomib, melphalan, and prednisone versus bortezomib, thalidomide, and prednisone as induction therapy followed by maintenance treatment with bortezomib and thalidomide versus bortezomib and prednisone in elderly patients with untreated multiple myeloma: a randomised trial. Lancet Oncol. 2010;11(10):934-941.

21. Moreau P, Avet-Loiseau H, Facon T, et al. Bortezomib plus dexamethasone versus reduced-dose bortezomib, thalidomide plus dexamethasone as induction treatment before autologous stem cell transplantation in newly diagnosed multiple myeloma. Blood. 2011;118(22):5752-5758. quiz 982.

22. Niesvizky R, Flinn IW, Rifkin R, et al. Community-based phase IIIB trial of three UPFRONT bortezomib-based myeloma regimens. J Clin Oncol. 2015;33(33):3921-3929.

23. Blade J, Rosinol L, Cibeira MT. Prognostic factors for multiple myeloma in the era of novel agents. Ann Oncol. 2008;19(suppl 7): vii117-vii120.
24. Chim CS, Sim J, Tam S, Tse E, Lie AK, Kwong YL. LDH is an adverse prognostic factor independent of ISS in transplant-eligible myeloma patients receiving bortezomib-based induction regimens. Eur J Haematol. 2015;94(4):330-335.

25. Morawska M, Grzasko N, Kostyra M, Wojciechowicz J, Hus M. Therapy-related peripheral neuropathy in multiple myeloma patients. Hematol Oncol. Epub 2014 Nov 14.

26. Koh Y, Lee SY, Kim I, et al. Bortezomib-associated peripheral neuropathy requiring medical treatment is decreased by administering the medication by subcutaneous injection in Korean multiple myeloma patients. Cancer Chemother Pharmacol. 2014;74(3):653-657.

27. Bringhen S, Larocca A, Rossi D, et al. Efficacy and safety of onceweekly bortezomib in multiple myeloma patients. Blood. 2010;116(23): 4745-4753.

28. Reeder CB, Reece DE, Kukreti V, et al. Once- versus twice-weekly bortezomib induction therapy with CyBorD in newly diagnosed multiple myeloma. Blood. 2010;115(16):3416-3417.

29. Moreau P, Pylypenko H, Grosicki S, et al. Subcutaneous versus intravenous administration of bortezomib in patients with relapsed multiple myeloma: a randomised, phase 3, non-inferiority study. Lancet Oncol. 2011;12(5):431-440.

30. Kim SJ, Kim K, Kim BS, et al. Bortezomib and the increased incidence of herpes zoster in patients with multiple myeloma. Clin Lymphoma Myeloma. 2008;8(4):237-240.

31. Chanan-Khan A, Sonneveld P, Schuster MW, et al. Analysis of herpes zoster events among bortezomib-treated patients in the phase III APEX study. J Clin Oncol. 2008;26(29):4784-4790.
OncoTargets and Therapy

\section{Publish your work in this journal}

OncoTargets and Therapy is an international, peer-reviewed, open access journal focusing on the pathological basis of all cancers, potential targets for therapy and treatment protocols employed to improve the management of cancer patients. The journal also focuses on the impact of management programs and new therapeutic agents and protocols on

\section{Dovepress}

patient perspectives such as quality of life, adherence and satisfaction. The manuscript management system is completely online and includes a very quick and fair peer-review system, which is all easy to use. Visit http://www.dovepress.com/testimonials.php to read real quotes from published authors. 